Annuaire suisse de politique de développement

18 | 1999

La Suisse et l'action humanitaire

\title{
7. Politique économique extérieure
}

\section{Gérard Perroulaz}

\section{OpenEdition}

Journals

Édition électronique

URL : http://journals.openedition.org/aspd/683

DOI : $10.4000 /$ aspd.683

ISSN : 1663-9669

\section{Éditeur}

Institut de hautes études internationales et du développement

\section{Édition imprimée}

Date de publication : 1 janvier 1999

Pagination : 199-218

ISSN : 1660-5934

\section{Référence électronique}

Gérard Perroulaz, «7. Politique économique extérieure », Annuaire suisse de politique de développement [En ligne], 18| 1999, mis en ligne le 17 juillet 2012, consulté le 08 septembre 2020. URL : http:// journals.openedition.org/aspd/683; DOI : https://doi.org/10.4000/aspd.683 


\section{POLITIQUE ÉCONOMIQUE EXTÉRIEURE*}

D ANS CE CHAPITRE sont détaillés certains instruments autonomes de la politique économique extérieure de la Suisse (GRE, préférences douanières), ainsi que les relations bilatérales avec certains pays en développement ou pays de l'Europe centrale et orientale (accords économiques bilatéraux, visites de chefs d'Etat en Suisse, délégations suisses menées par des conseillers fédéraux). La politique de la Suisse auprès des organisations multilatérales est examinée dans les chapitres concernant ces organisations (FMI, Banque mondiale, CNUCED, OMC, banques régionales de développement).

\subsection{INSTRUMENTS DE LA POLITIQUE ÉCONOMIQUE EXTÉRIEURE}

La politique économique extérieure comprend les mesures de la Confédération pour favoriser les relations économiques avec l'étranger et protéger les intérêts des exportateurs et investisseurs suisses dans le monde. La Confédération dispose de moyens variés pour défendre les intérêts économiques suisses à l'étranger, avec plus de 300 diplomates en fonction, les chambres de commerce présentes dans certains pays partenaires, l'ensemble des accords bilatéraux, la participation aux grandes conférences internationales et aux travaux des organisations multilatérales (avec le rôle important pris par l'OMC ces dernières années). Pour protéger les intérêts des investisseurs suisses à l'étranger, la Confédération accorde une grande importance à la conclusion d'accords bilatéraux de promotion et de protection des investissements (86 accords conclus jusqu'à ce jour), ainsi que de conventions de double imposition. Les accords économiques concernent en premier lieu les principaux pays partenaires économiques de la Suisse, mais aussi les nouveaux marchés émergents ou potentiels. Le tableau n ${ }^{\circ}$ 1.1.C.2 de la partie «Statistiques» du présent Annuaire donne une liste des principaux pays en développement partenaires commerciaux de la Suisse. La balance commerciale avec ces pays est souvent très excédentaire pour la Suisse (celle-ci exporte généralement bien plus qu'elle n'importe des pays en développement). Les investissements directs étrangers de la Suisse se concentrent sur quelques pays en développement les plus avancés. En 1996, les principaux pays en développement où se concentraient les investissements directs étaient les suivants: Brésil, Hongkong, Mexique, Chili et Singapour.

L'Office fédéral des affaires économiques extérieures (OFAEE), relevant du Département fédéral de l'économie (DFE), s'occupe des dossiers de la politique économique extérieure de la Suisse. Il cherche à faciliter l'accès aux marchés et l'intégration économique. Il apporte un appui à la promotion des exportations et contribue à l'amélioration des conditions-cadres pour la compétitivité de la place économique suisse. Cet office assume aussi une partie des tâches de coopération au développement et de coopération avec les pays de l'Europe centrale et orien-

* Par Gérard Perroulaz, chargé de recherche à l'IUED. 
tale (voir chapitres 8 , partie 8.4 et 9 de la «Revue»). Le Conseil fédéral a décidé en mai 1998 de procéder à une fusion de l'OFAEE et de l'Office fédéral du développement économique et de l'emploi (OFDE, anciennement OFIAMT), afin de rassembler sous un même toit toutes les compétences du domaine économique. Les aspects de politiques économiques intérieure et extérieure seront ainsi intégrés dès le départ dans les processus de décision. Le nouvel office, qui deviendra opérationnel d'ici l'an 2000, travaillera dans les domaines de la coopération au développement et de l'aide aux pays de l'Europe de l'Est, de la politique économique générale, de la politique en faveur de la place économique, du marché du travail, de l'assurance chômage et de la protection des travailleurs.

\subsection{GARANTIE CONTRE LES RISQUES À L'EXPORTATION}

La Garantie contre les risques à l'exportation (GRE) est un instrument pour faciliter la conclusion de contrats à l'étranger, dans les pays où l'exportateur hésiterait à prendre le risque de ne pas être payé. La nouvelle ordonnance sur la GRE est entrée en vigueur le $1^{\text {er }}$ juillet 1998. Les émoluments (primes d'assurance) ont été adaptés pour se conformer à l'arrangement conclu entre les pays de l'OCDE. La nouvelle ordonnance fixe les émoluments selon une échelle de risque allant de un à sept, les pays acheteurs étant classés dans une de ces sept catégories, en fonction du risque encouru. Fin 1997, le total des engagements de la GRE atteignait 6,4 milliards de francs, alors que les nouvelles garanties accordées pendant l'année 1997 se sont élevées à 2,5 milliards de francs. Le Moyen-Orient et les autres pays de l'Asie représentent plus de $61 \%$ des engagements totaux.

\section{$\square$ Fonctionnement général de la GRE}

La GRE permet aux exportateurs suisses de se protéger contre le non-paiement de livraisons. La Confédération met cet instrument à la disposition de l'exportateur suisse pour lui faciliter la conclusion de contrats de vente à l'étranger. La GRE couvre la livraison ou la location de marchandises, la construction d'ouvrages, l'exécution des travaux de construction, la remise de licences sur des biens immatériels, la prestation de services de conseils scientifiques, techniques et économiques. Les bénéficiaires de la garantie sont donc non seulement l'exportateur de marchandises, mais aussi le constructeur d'ouvrages ou le bureau d'ingénieurs. Cette assurance est très appréciée par l'exportateur lorsque des difficultés de recouvrement sont à craindre en raison de la situation politiquement et économiquement instable dans le pays de l'acheteur.

Une commission de huit membres nommés par le Conseil fédéral examine les demandes de garantie déposées par les exportateurs suisses auprès du bureau pour la GRE (à Zurich)'. Sur la base des propositions de la commission, c'est l'OFAEE qui décide de l'octroi de la GRE lorsque le montant n'excède pas 5 millions de francs. Les garanties supérieures à 5 millions de francs ou politique-

1. En 1998, la commission comprenait quatre représentants de la Confédération (OFAEE, DDC, Administration des finances, Office fédéral du développement économique et de l'emploi (OFDE, anciennement OFIAMT), trois représentants de l'économie privée (Union suisse du commerce et de l'industrie - Vorort, Société suisse des constructeurs de machines, Société suisse des industries chimiques) et une représentante des milieux syndicaux (Syndicat chrétien de l'industrie, de l'artisanat et des services). La commission est présidée par l'ambassadeur Rolf M. Jeker (OFAEE). 
ment délicates doivent avoir l'aval du Conseil fédéral. Les garanties supérieures à 10 millions de francs doivent en plus recevoir le consentement du Département fédéral des finances.

Le résultat financier global de la GRE est bénéficiaire depuis quelques années, ce qui permet à la GRE de rembourser à la Confédération depuis 1995 une partie du prêt que celle-ci lui avait accordé pour son fonctionnement; 22 millions de francs d'intérêts ont été ainsi été payés à la Confédération et la dette réduite de 250 millions de francs (l'avance de la Confédération s'élevant encore à 1,34 milliard de francs). S'il est vrai que les affaires de la GRE dégagent un bénéfice annuel ces dernières années, il faut préciser que le résultat annuel s'est soldé par une perte pour treize ans sur les vingt-quatre dernières années (résultat bénéficiaire pour neuf ans et équilibre des comptes pour deux ans). Les dédommagements versés par la GRE aux exportateurs sinistrés en 1997 se sont élevés à 53 millions de francs (108 millions de francs en 1996).

\section{Risques couverts par la GRE}

La GRE couvre l'exportateur de certains risques encourus dans ses contrats avec l'étranger que ni luimême ni l'acheteur étranger ne sont en mesure d'influencer. II s'agit des risques suivants:

- Risque politique - Evénements politiques (guerre, révolution, émeutes...) qui empêchent l'acheteur d'effectuer les paiements convenus ou aboutissent à la perte de la marchandise dont l'exportateur est encore propriétaire.

ป Risque de transfert - Les paiements de l'acheteur ne sont pas transférés en raison de mesures gouvernementales de contrôle des changes ou de rééchelonnement de la dette extérieure du pays.

- Risque de fabrication - Si une marchandise n'est pas livrée suite à des événements politiques ou à des mesures gouvernementales.

ـ Risque de ducroire (risque commercial) - Insolvabilité ou refus de payer d'acheteurs de droit public ou de sociétés d'utilité publique. Sur demande de l'exportateur, ce risque peut être couvert pour des acheteurs privés, lorsque ceux-ci bénéficient de la garantie de paiement d'une banque acceptée par la GRE ou de l'Etat.

\ Risque secondaire des contrats libellés en monnaie étrangère - L'exportateur peut, contre paiement d'un supplément de prime, faire calculer l'indemnisation en francs suisses, selon le taux de change en vigueur au moment du sinistre. Ce n'est pas une garantie sur les taux de change, mais cette couverture garantit uniquement que l'exportateur ne soit pas sous-assuré en raison de fluctuations de change.

La GRE ne couvre pas le risque d'insolvabilité d'un acheteur privé, ni les risques pouvant être couverts par des assurances privées (risques de transport par exemple), ni les risques monétaires ou les fluctuations de taux de change. La garantie contre les risques monétaires avait dû être supprimée en 1985, en raison des grandes pertes qu'elle avait provoquées.

Source: bureau pour la GRE, Zurich.

\section{$\square$ Nouvelle ordonnance sur la GRE}

Un accord a été conclu au sein de l'OCDE pour harmoniser les primes fixées par les assureurs étatiques des exportations. L'émolument représente le prix de la couverture (prime d'assurance) que l'exportateur doit payer pour être couvert par la Garantie contre les risques à l'exportation. L'arrangement relatif aux crédits à 
l'exportation a été adopté le 20 juin 1997 par les ministres de l'OCDE. Il règle les émoluments minimaux à appliquer aux garanties de l'Etat fournies pour des crédits à l'exportation dont le terme est supérieur à deux ans. Les émoluments reposent sur une classification commune des risques des pays acheteurs, dans le cadre d'une échelle de risque allant de un à sept. Cette classification est revue périodiquement d'entente entre les experts des pays membres de l'OCDE, en se fondant sur les données macroéconomiques de chaque pays considéré, ainsi que sur les expériences acquises en matière de paiement par les différents assureurs. Les pays doivent ainsi appliquer un émolument minimum.

La Suisse s'est déclarée favorable à l'arrangement de l'OCDE qui permet une meilleure discipline entre pays. La concurrence entre entreprises reposera ainsi sur le plan technique et les prix compétitifs plutôt que sur les différences dans les systèmes de subventions aux exportations. L'application d'une prime plus ou moins grande d'un pays à l'autre entraîne en effet une distorsion dans la concurrence, une entreprise d'un pays donné pouvant ainsi bénéficier d'un soutien étatique plus important qu'une autre entreprise d'un autre pays (les distorsions peuvent même exister entre une entreprise d'un pays et une filiale de la même entreprise à l'étranger).

L'arrangement de l'OCDE entre en vigueur le $1^{\text {er }}$ avril 1999, après une période transitoire pour adapter les différents systèmes nationaux de GRE. En Suisse, la nouvelle ordonnance sur la GRE, avec révision des émoluments, est entrée en vigueur le $1^{\text {er }}$ juillet $1998^{2}$. La nouvelle ordonnance suisse fixe ainsi les primes de base, qui augmentent en fonction de la durée de la garantie et selon les sept catégories de pays. Par exemple, une entreprise exportant pour un montant assuré d'un million de francs devra payer une prime de base variant de 8500 francs à $74^{\prime} 000$ francs selon que le pays acheteur est réputé peu ou très risqué (si la garantie dure cinq ans). Des émoluments supplémentaires peuvent être perçus pour les risques non couverts par l'assurance de base (inclusion du risque monétaire par exemple ${ }^{3}$ ). Des rabais sur les primes peuvent être aussi accordés en cas de diminution du risque (comme lors de participation d'institutions de financement internationales, ou lors de livraisons dans le cadre de l'aide financière bilatérale, part non publique des financements mixtes par exemple).

\section{$\square$ Nouvelles garanties accordées en 1997}

Selon la deuxième colonne du tableau $\mathrm{n}^{\circ} 15$, le montant total des nouvelles garanties accordées s'est élevé à 2,5 milliards de francs en 1997, soit une augmentation de $74 \%$ par rapport à l'année précédente ( 1,4 milliard) ; 87\% des nouvelles garanties ont été accordées aux pays suivants (du plus grand au plus petit): Turquie (plus de 700 millions de francs de nouvelles garanties), Chine (plus de 400 millions), Bahreïn, Iran, Arabie Saoudite, Mexique, Maroc, Israël, Pologne, Brésil; $83 \%$ des nouvelles garanties concernent la branche de l'industrie des machines et $14 \%$ la chimie. Les six projets les plus importants (de plus de 50 millions de francs chacun) représentaient $39 \%$ du volume des nouvelles garanties.

2. «Ordonnance sur la Garantie contre les risques à l'exportation» du 15 juin 1998, Recueil officiel des lois fédérales, n 26, 7.7.98, pp. 1624-1642. Cette ordonnance remplace celle du 15 janvier 1969.

3. Depuis juillet 1996, l'exportateur peut, contre paiement d'un supplément de prime, faire calculer l'indemnisation en francs suisses, selon le taux de change au moment du sinistre. 


\section{Barrage des Trois-Gorges (Chine)}

L'un des plus grands projets accordés par la GRE en 1997 est l'exportation de matériel pour le barrage des Trois-Gorges en Chine (pour l'entreprise ABB Asea Brown Boveri). ABB a obtenu une commande de 14 générateurs pour un montant de 340 millions de francs. Les campagnes de la Déclaration de Berne et de la Communauté de travail des œuvres d'entraide se sont focalisées en 1996 et 1997 sur l'opposition à l'octroi de la GRE aux livraisons suisses pour la construction du barrage des Trois-Gorges; 25'000 cartes postales préparées par la Déclaration de Berne ont été envoyées au Conseil fédéral et à $\mathrm{ABB}$ pour leur demander de renoncer à l'octroi de la GRE pour ce projet controversé de grand barrage. Ces campagnes réclamaient d'autre part une réforme du fonctionnement de la GRE (prise en compte des objectifs de développement durable et des droits de l'homme dans l'octroi de la GRE, élargissement de la commission de la GRE à un représentant des œuvres d'entraide, meilleure transparence sur les garanties accordées).

ASTM 1998, pp. 281-282: détail des campagnes contre le projet du barrage des Trois-Gorges.

\section{Barrage Ilisu (Turquie)}

En novembre 1998, la Déclaration de Berne, le WWF et les œuvres d'entraide se sont adressés au Conseil fédéral afin de lui demander de refuser à $\mathrm{ABB}$ et Sulzer la GRE destinée à couvrir l'exportation pour le chantier d'irrigation sur le Tigre et l'Euphrate en Turquie (barrage d'Ilisu, dans le cadre du projet dénommé GAP, projet du Sud-Est anatolien). Les deux entreprises suisses ont sollicité une garantie pour un montant de 470 millions de francs. Plusieurs arguments ont été avancés par les ONG pour demander le refus de l'octroi de la GRE à ce grand projet jugé non conforme à une politique plus cohérente de la Suisse envers les pays en développement. Premièrement, le chantier viendrait bouleverser les données politiques et sociales dans une région kurde de la Turquie. Les déplacements de population pourraient alimenter les flux de personnes allant dans les bidonvilles des grandes villes turques et le flux de réfugiés kurdes en Suisse; 15'000 à 20 '000 personnes seraient déplacées par ce projet et l'obtention de dédommagements et de conditions adéquates du déplacement de population n'est pas assurée dans un régime réputé répressif. Deuxièmement, en octroyant la GRE, la Suisse apporterait son soutien indirect à la politique turque jugée agressive par les pays voisins du Sud (Irak et Syrie). Par le potentiel de réserve énorme du lac de retenue du barrage, le projet GAP pourrait en effet représenter une menace sur l'approvisionnement en eau pour ces pays en aval des fleuves du Tigre et de l'Euphrate. La Turquie n'a d'ailleurs pas adhéré à la Convention des Nations Unies sur l'utilisation des voies maritimes internationales, qui devrait régler les conflits transfrontaliers dans l'utilisation de l'eau. Le troisième point concerne l'impact du barrage sur l'environnement et la santé (propagation de la malaria). Le projet ne respecterait en outre pas les lignes directrices de la Banque mondiale, qui d'ailleurs ne soutient pas ce projet financièrement. Finalement, la construction représente aussi une menace pour certains sites et biens culturels.

Le Conseil fédéral a décidé fin novembre 1998 de donner son aval à l'octroi de la GRE pour des commandes d'une valeur de 470 millions de francs pour le barrage d'Ilisu (et de 285 millions de francs pour la centrale au gaz d'Ankara). Le Conseil fédéral motive cette décision positive pour le projet du barrage d'Ilisu par la reconnaissance des besoins énergétiques de la Turquie, par l'importance de 
ces projets pour l'emploi en Suisse et par certaines garanties données par la Turquie concernant le dédommagement des personnes déplacées. La commission de la GRE a entendu l'avis d'ONG avant de recommander l'acceptation du projet et, pour la première fois, des consultations en vue d'une coordination internationale ont eu lieu entre les sept agences de garanties à l'exportation des autres pays où des demandes d'octroi avaient été formulées par des entreprises d'exportation. Le Conseil fédéral a chargé le Département de l'économie d'assurer, d'entente avec les autres pays concernés, un dialogue avec les responsables de la construction du barrage et de mettre sur pied une instance de contrôle indépendante (monitoring) pour s'assurer que les déplacements de population s'effectuent selon des standards acceptables sur le plan international.

Tableau no 15: Répartition géographique des nouvelles garanties et du total des engagements de la GRE en 1997

\begin{tabular}{lrrrr}
\hline & $\begin{array}{c}\text { Nouvelles garanties } \\
\text { en 1997 }\end{array}$ & \multicolumn{2}{c}{$\begin{array}{c}\text { Total des engagements } \\
\text { au 31.12.1997 }\end{array}$} \\
\hline Groupes de pays & $\begin{array}{c}\text { Millions } \\
\text { de fr. }\end{array}$ & $\begin{array}{r}\text { part } \\
\text { en } \%\end{array}$ & $\begin{array}{r}\text { Millions } \\
\text { de fr. }\end{array}$ & $\begin{array}{r}\text { part } \\
\text { en \% }\end{array}$ \\
\hline Europe (sans Europe cent. et orient.) & 755.6 & 30.5 & 1128.8 & 17.6 \\
\hline Pays d'Europe centrale et orientale & 118.5 & 4.8 & 195.3 & 3.0 \\
\hline Amérique centrale et du Sud & 164.7 & 6.6 & 646.9 & 10.1 \\
\hline Afrique & 147.9 & 6.0 & 509.9 & 7.9 \\
\hline Moyen-Orient & 713.5 & 28.8 & 1544.1 & 24.0 \\
\hline Asie & 579.4 & 23.4 & 2382.8 & 37.1 \\
\hline Australie & 0.0 & 0.0 & 12.9 & 0.2 \\
\hline Total & $\mathbf{2 4 7 9 . 6}$ & $\mathbf{1 0 0 . 0}$ & $\mathbf{6 4 2 0 . 7}$ & $\mathbf{1 0 0 . 0}$ \\
\hline dont: 70 pays les plus défavorisés & 550.7 & 22.2 & 1186.3 & 118.5 \\
\hline \multicolumn{1}{c}{ Antrique } & 0.0 & 0.0 & 2.5 & 0.0 \\
\hline \multicolumn{1}{c}{ Asie } & 18.1 & 0.7 & 125.2 & 1.9 \\
\hline
\end{tabular}

Source: Rapport annuel 1997 de la GRE - Juin 1997, tableau de I'annexe C/1.

$\square$ Total des engagements de la GRE

Le total des engagements s'élevait à 6,4 milliards de francs fin 1997 (troisième colonne du tableau $n^{\circ} 15$ ). Le graphique $n^{\circ} 4$ relève les 15 principaux pays bénéficiaires, qui représentent $83,7 \%$ du total des engagements de la GRE. L'Indonésie, la Turquie, la Chine et l'Iran représentent ensemble 53\% de tous les engagements.

L'engagement total a fortement augmenté de fin 1996 à fin 1997 pour la Turquie (passant de 531 millions de francs fin 1996 à plus d'un milliard de francs fin 1997), la Chine (passant de 372 millions à 680 millions) ainsi que Bahreïn ( de 49 millions à 214 millions). Avec les nouveaux engagements de la GRE en 1998, la Turquie a même devancé l'Indonésie, prenant ainsi la première place des 15 principaux pays bénéficiaires de la garantie.

Les caractéristiques principales de la répartition géographique des engagements de la GRE sont les suivantes (situation fin 1997):

\section{Europe}

Les pays européens représentent 17,6\% des engagements de la GRE, soit plus de 1,1 milliard de francs. Mais ces engagements concernent principalement la Turquie (pour plus d'un milliard de francs). 


\section{Pays d'Europe centrale et orientale}

Les engagements vers cette région ne s'élèvent qu'à 195 millions de francs, soit $3 \%$ des engagements totaux, principalement pour couvrir des exportations vers la Pologne (67 millions de francs fin 1997) et la Roumanie (64 millions). Il faut toutefois préciser qu'il existe un instrument de garanties complémentaires sur les crédits d'aide aux pays de l'Europe centrale et orientale. Ces garanties sont octroyées pour les pays où la GRE n'est pas (ou seulement partiellement) disponible, en raison d'un risque jugé trop élevé (voir chapitre 9 pour les instrument de l'aide publique à ces pays). Les engagements envers la Russie sont assez faibles (6 millions de francs fin 1997).

\section{Moyen-Orient}

Le Moyen-Orient représente le quart du total des engagements, surtout pour des exportations vers l'Iran, l'Arabie Saoudite, Israël, Bahreïn et la Jordanie.

Autres pays de l'Asie

La part relative de l'Asie est importante (37\% des engagements totaux, plus 24\% si l'on inclut les pays du Moyen-Orient).

Parmi les pays asiatiques frappés par la crise économique et financière, la GRE est très fortement engagée en Indonésie (pour 1,1 milliard de francs), et dans une bien moindre mesure aux Philippines (116 millions de francs), en Thaïlande (69 millions) et en Corée du Sud (4,5 millions). Elle n'a aucun engagement au Japon et en Malaisie. Conséquence de la crise et de la baisse de confiance auprès de certains exportateurs, la GRE risque dans l'avenir d'être sollicitée davantage pour certains contrats dans ces pays (contrats qui jusqu'ici se finançaient sans faire appel à la GRE puisqu'ils étaient réputés peu risqués). La GRE a déclaré en été 1998 rester sereine face aux risques de défaut de paiement des considérables exportations couvertes vers l'Indonésie (notamment en raison de ses importantes provisions pour faire face à des coups durs).

Amérique centrale et du Sud

L'Amérique centrale et du Sud représente 10\% de tous les engagements (647 millions de francs), principalement pour couvrir des exportations vers le Brésil (214 millions), le Mexique (176 millions), l'Argentine (101 millions) et la Colombie (60 millions).

\section{Afrique}

Seuls $8 \%$ des garanties couvertes pour les exportations suisses l'étaient pour le continent africain (soit 510 millions de francs). Les principaux pays sont l'Algérie (104 millions), l'Egypte (99 millions), le Maroc (93 millions), l'Afrique du Sud (65 millions de francs) et le Zimbabwe (45 millions). 


\section{Graphique $n^{\circ} 4$ : Les 15 principaux pays couverts par la GRE}

Montant total des engagements au 31.12.97, en millions de francs

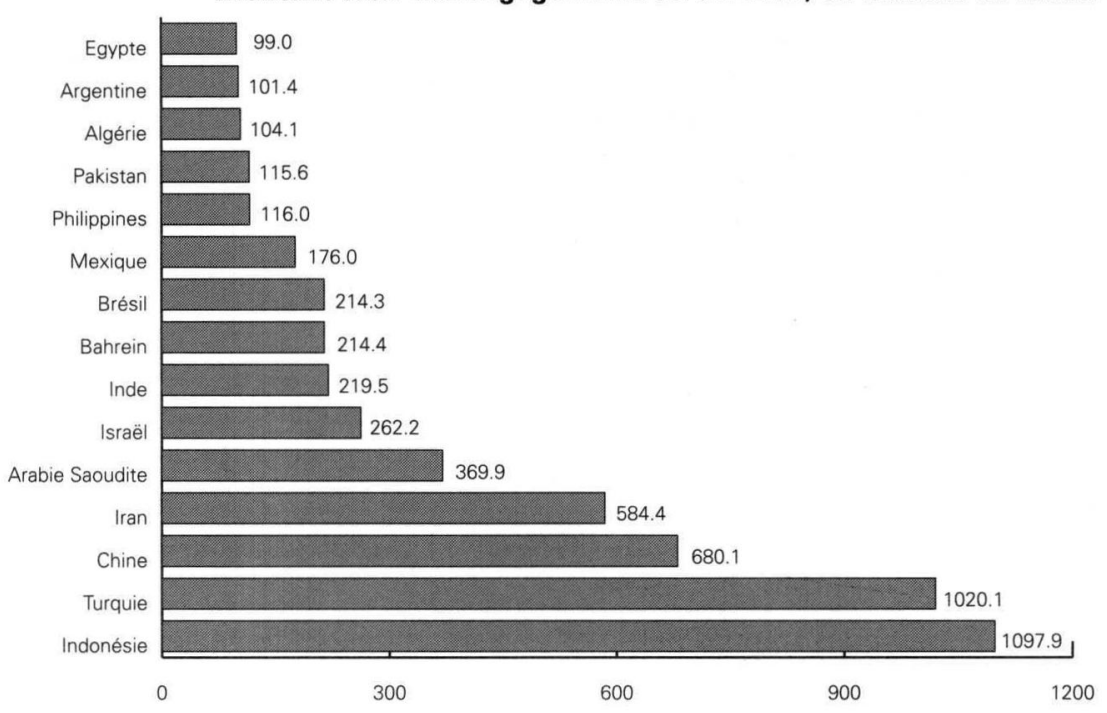

Source: Rapport annuel 1997 de la GRE, juin 1998, graphique à partir des tableaux de l'annexe G/1 à G/3.

\section{$\square$ Pays en développement les plus défavorisés}

En 1980, le Parlement avait ajouté la disposition suivante à la loi sur la GRE (art. ${ }^{\text {er }}$, al. 2): «S'agissant d'exportations à destination des pays en développement les plus défavorisés, la Suisse tiendra compte des principes fondamentaux de la politique suisse en matière d'aide au développement.» La liste des 70 pays les plus défavorisés définie par la Confédération comprend les 48 pays les moins avancés (PMA) et 22 autres pays à bas revenus ${ }^{4}$ (pays disposant d'un PNB par habitant inférieur à 765 dollars en 1996). Entre 1996 et 1997, l'Albanie et la Géorgie ont été retirées de la liste des pays les plus défavorisés.

En 1997, 96,7\% des nouvelles garanties pour les pays les plus défavorisés concernaient le continent asiatique. Le total des engagements de la GRE pour ces pays atteignait 1186,3 millions de francs en 1997 , ce qui représente $18,5 \%$ du total des engagements pour tous les pays; $89 \%$ du total des engagements pour ce groupe de pays concernaient l'Asie (et 10,6\% l'Afrique).

\section{$\square$ Accords de consolidation de dettes}

Fin 1997, 90 accords bilatéraux de rééchelonnement de dettes étaient en vigueur pour 31 pays. Les avoirs de la GRE issus de ces accords ont atteint 3,1 milliards de francs (y compris les intérêts capitalisés et intérêts échus, mais non payés). Ces mesures de désendettement permettent à la GRE de céder à la Confédération les créances consolidées.

Six nouveaux accords bilatéraux de rééchelonnement ont été conclus par la Confédération avec cinq pays: Cameroun, Guinée-Conakry (deux accords), Jor-

4. La liste des 70 pays les plus défavorisés comprend les 48 pays les moins avancés (PMA), ainsi que les pays suivants: Arménie, Azerbaïdjan, Bosnie, Cameroun, Chine, Congo, Côte d'Ivoire, Ghana, Guyane, Honduras, Inde, Kenya, Kirghizistan, Mongolie, Nicaragua, Nigeria, Pakistan, Sénégal, Sri Lanka, Tadjikistan, Vietnam, Zimbabwe. 
danie, Madagascar et Russie. Le montant total rééchelonné s'élève à 425,5 millions de francs. Le rapport annuel de la GRE indique que la volonté et la capacité de paiement des pays débiteurs s'améliorent ces dernières années: $81 \%$ des remboursements en capital dus et $95 \%$ des intérêts demandés ont été ainsi effectivement payés en 1997 (taux respectivement de $82 \%$ et $84 \%$ en 1996).

[Q ASTM 1999, section 1.2 (mesures de désendettement prises par la Suisse) du premier chapitre («Relations financières internationales) de la partie «Revue».

\section{SOURCES}

Bureau pour la GRE, OFAEE, Rapport annuel 1997 de la GRE, Zurich et Berne, juin 1998

«Ordonnance sur la Garantie contre les risques à l'exportation» du 15 juin 1998, Recueil officiel des lois fédérales, n²6, 7.7.98, pp. 1624-1642.

Communiqué de presse du Département fédéral de l'économie, Service de presse et d'information, Berne, 22.6.98.

Le Temps, 23.6.98.

Neue Zürcher Zeitung, «Noch keine Grippesymptome bei der ERG», 23.6.98; «Umstrittene ERG für Kraftwerk in der Türkei», 25.11.98; «Exportrisikogarantie für türkische Kraftwerke», 1.12.98.

Tribune de Genève, «Ilisu, un projet de barrage turc qui embarrasse la Suisse», 25.12.98

Déclaration de Berne, site Internet http://www.access.ch/evb/db

ADRESSE

Bureau pour la garantie contre les risques à l'exportation, Kirchenweg 8, 8032 Zurich.

SITE INTERNET

GRE: http://www.swiss-erg.com/

\subsection{PRÉFÉRENCES DOUANIÈRES}

La Suisse accorde depuis 1972 des droits de douane préférentiels sur l'importation de certains produits en provenance de pays en développement. Les objectifs et les principes communs des préférences douanières ont été conclus sur le plan multilatéral à la CNUCED. Les schémas nationaux sont revus périodiquement selon ces principes. En septembre 1996, le Parlement avait accepté une révision du schéma de préférences. Sur cette base, l'ordonnance fixant les droits de douane préférentiels est entrée en vigueur dès le $1^{\text {er }}$ mars 1997. L'Annuaire 1998 (pp. 284-289) détaillait la teneur de cette nouvelle ordonnance, ainsi que l'utilisation des préférences de 1994 à 1996. Parmi les changements principaux du nouveau schéma de préférences tarifaires, on peut relever l'introduction du «principe de graduation intégrale» selon le stade de développement atteint par le pays partenaire. La Suisse peut selon ce principe supprimer les préférences accordées aux pays en développement les plus avancés. Dès le $1^{\text {er }}$ mars 1998, la Suisse a ainsi retiré les préférences à certains pays (dont la Corée du Sud, Hongkong, le Mexique et Singapour). Le principe de "graduation partielle», qui existait déjà auparavant, permet en outre à la Suisse de supprimer les préférences douanières pour des produits spécifiques exportés par certains pays. La Suisse n'octroie par exemple pas de réductions tarifaires pour les textiles en provenance de la Chine et de Macao.

\section{$\square$ Préférences douanières de 1995 à 1997}

Le tableau suivant présente les montants des importations de la Suisse ayant bénéficié des préférences ces dernières années. Trois quarts environ des importations en provenance des pays en développement pourraient bénéficier des préférences (soit $6 \%$ à $8 \%$ environ du volume total des importations suisses). Le 
recours effectif à ces préférences est cependant relativement faible, puisqu'en moyenne seule la moitié des avantages tarifaires qui pourraient être accordés est réellement utilisée (47\% en 1997). Le degré d'utilisation des avantages accordés est en fait très élevé pour les produits agricoles, alors qu'il est plus bas pour les produits industriels. La marge de préférence étant faible pour certains produits industriels, les exportateurs hésitent à faire les démarches nécessaires qui leur permettraient de bénéficier de ces avantages. Les importations ayant effectivement bénéficié des préférences se sont élevées en 1997 à 2,6 milliards de francs, dont 340 millions de francs d'importations avec des droits de douane nuls.

Tableau n`16: Importations suisses et préférences tarifaires 1995-1997

\begin{tabular}{|c|c|c|c|}
\hline Importations et préférences & 1995 & 1996 & 1997 \\
\hline $\begin{array}{l}\text { 1. Volume total des importations de la Suisse, } \\
\text { en mio. de francs }\end{array}$ & 94483 & 96664 & 110087 \\
\hline $\begin{array}{l}\text { 2. Importations en provenance des pays en } \\
\text { développement (PVD), en mio. de francs }\end{array}$ & 6175 & 5828 & 8318 \\
\hline $\begin{array}{l}\text { 3. Part des importations en provenance des PVD } \\
\text { en } \% \text { (point } 2 \text { en } \% \text { du point } 1 \text { ) }\end{array}$ & $6.5 \%$ & $6.0 \%$ & $7.6 \%$ \\
\hline $\begin{array}{l}\text { 4. Importations pouvant bénéficier du } \\
\text { traitement préférentiel, en mio. de francs }\end{array}$ & 4223 & 4326 & 5633 \\
\hline $\begin{array}{l}\text { 5. Importations ayant effectivement bénéficié des } \\
\text { préférences, en mio. de francs }\end{array}$ & 2188 & 2204 & 2649 \\
\hline $\begin{array}{l}\text { 6. Degré d'utilisation des avantages accordés } \\
\text { en } \% \text { (point } 5 \text { en } \% \text { du point } 4 \text { ) }\end{array}$ & $52 \%$ & $51 \%$ & $47 \%$ \\
\hline
\end{tabular}

Source: OFAEE.

\subsection{PROMOTION DES EXPORTATIONS}

La Confédération soutient financièrement l'Office suisse d'expansion commerciale (OSEC) - pour un montant de près de 10 millions de francs en 1997 - et d'autres organisations mettant sur pied des actions de promotion des exportations (chambres de commerce suisses à l'étranger par exemple). L'OSEC est l'institution officielle suisse de promotion du commerce extérieur (subventionnée par la Confédération pour un tiers de son budget). Ses tâches recouvrent le développement des relations économiques extérieures et la propagation de l'image de l'économie suisse dans le monde. Il doit aider les exportateurs à trouver de nouveaux débouchés sur les marchés internationaux. L'office fournit des conseils et des informations sur les marchés et sur l'économie suisse d'exportation ${ }^{5}$; il participe aussi régulièrement à des foires économiques.

Le Conseil fédéral prépare une nouvelle loi sur la promotion du commerce extérieur. L'idée principale est de conclure un accord de prestations de services entre la Confédération et une société de management. Le soutien qui était accordé jusqu'à maintenant soit à l'OSEC, aux chambres de commerce ou aux associations professionnelles pourrait à l'avenir être transféré à une société de management. Celle-ci travaillera sur la base des objectifs fixés avec l'OFAEE et sera indemni-

5. OSEC, 4, avenue de l'Avant-Poste, 1001 Lausanne. Tél. 021/320.32.31, fax 021/320.73.37, site Internet: http://www.osec.ch. 
sée par l'aide financière de la Confédération. Cette aide sera cependant mieux liée aux résultats du travail de la société ${ }^{6}$.

\subsection{ACCORDS ÉCONOMIQUES BILATÉRAUX}

\section{Accords de promotion et de protection des investissements}

La Suisse fait partie des pays qui ont tissé un réseau très dense d'accords bilatéraux concernant la protection et l'encouragement des investissements. Un accord de protection des investissements englobe toutes les catégories d'avoirs dans l'autre pays: propriété de biens et d'immeubles, actions, parts sociales et autres formes de participation dans des sociétés, concessions, droits d'auteur, droits de propriété industrielle (brevets, dessins ou modèles industriels, marques, noms commerciaux). Chaque partie contractante s'engage à encourager, dans la mesure du possible, les investissements de l'autre partie contractante. Les principes de la nation la plus favorisée et de non-discrimination doivent être respectés. Aucun pays ne peut donc soumettre sur son territoire les investissements de l'autre pays à un traitement moins favorable que celui qu'il accorde aux investisseurs de tout Etat tiers (sauf à de rares exceptions, notamment lorsque le pays fait partie d'une zone de libre-échange). Le libre transfert des revenus des investissements doit être garanti (intérêts, dividendes, apports supplémentaires de capitaux pour le développement des investissements, produits de la liquidation partielle ou totale d'un investissement). Des mesures éventuelles d'expropriation ou de nationalisation doivent donner lieu au paiement d'indemnités adéquates. Les différends entre les parties contractantes ou entre un investisseur et l'Etat d'accueil donnent lieu à des consultations et peuvent être soumis à un tribunal arbitral.

Fin novembre 1998, la Suisse avait signé près de 90 accords de protection des investissements. Le tableau des pages 291 et 292 de l'Annuaire 1998 donnait la liste complète des accords conclus avec des pays en développement et des pays d'Europe centrale et orientale. Voici la liste des pays dont l'accord est entré en vigueur en 1997 ou 1998: Afrique du Sud, Cuba, Kazakhstan. D'autres accords avec les pays suivants ont été signés ces dernières années, mais ne sont pas encore (à fin novembre 1998) entrés en vigueur: Cambodge et Zimbabwe (accords signés en 1996), Inde, Mongolie, Philippines et Thaillande (accords signés en 1997), Arménie, Botswana, Emirats arabes unis, Ethiopie, île Maurice, Iran, Koweït (accords signés en 1998). Le tableau $n^{\circ} 17$ de ce chapitre donne la liste complète des accords en vigueur avec les pays de l'Europe centrale et orientale et les pays de la CEI.

\section{$\square$ Accords de double imposition}

Les pays signataires d'une convention de double imposition abandonnent une partie de leur souveraineté en matière fiscale afin d'éviter que des revenus ne soient imposés doublement, dans les deux pays concernés. Ces conventions bilatérales règlent l'imposition des personnes physiques et des entreprises: revenus, revenus immobiliers, dividendes, intérêts de créances, redevances, gains en capi-

6. OFAEE, Info@Bawi (bulletin), nº2, OFAEE, septembre 1998. 
tal, revenus d'artistes et sportifs, pensions et impôts sur la fortune (lorsque ces derniers existent dans les deux pays). Une fois signée par les deux Etats, la convention doit être approuvée par le Parlement. La décision n'est pas soumise au référendum facultatif.

L'Annuaire 1998 (p. 293) donnait la liste complète des pays avec lesquels la Suisse a signé une convention de double imposition. La Suisse a conclu jusqu'en 1998 plus de 50 conventions de ce type. Les conventions les plus récentes, depuis 1996, ont été signées avec les pays suivants: Argentine, République slovaque, Slovénie (1996), Thaïlande, Venezuela (signé en décembre 1996 et entré en vigueur au début de 1998), Vietnam (signé en 1996 et entré en vigueur le $1^{\text {er }}$ janvier 1998). Des conventions de double imposition sont en négociation avec plusieurs pays, dont les Emirats arabes unis.

\section{Accords de libre-échange}

Suite à l'élargissement de l'Union européenne, l'Association européenne de libre-échange (AELE) ne comprend depuis 1995 plus que les pays suivants: Islande, Liechtenstein, Norvège et Suisse. Les accords de libre-échange conclus avec des pays tiers ont pour but essentiel d'offrir aux entreprises exportatrices vers ces pays les mêmes conditions que celles dont bénéficient les entreprises de l'Union européenne. Les accords prévoient le libre-échange des produits industriels, des produits agricoles transformés ainsi que de certains produits agricoles non transformés (pour d'autres produits agricoles, droits de douane réduits). Ces accords contiennent aussi des dispositions sur le règlement des différends et sur la protection de la propriété intellectuelle. L'AELE s'efforce de conclure des accords avec les pays du sud du bassin méditerranéen, notamment suite à la décision de l'Union européenne de créer à terme une zone de libre-échange euroméditerranéenne (partenariat avec 11 pays riverains de la mer, conclu en 1995). L'Union européenne a déjà conclu des accords de libre-échange avec certains pays ou autorités de la région (Israël, Jordanie, Maroc, OLP, Tunisie) et des négociations sont en cours avec d'autres pays (Algérie, Egypte et Liban).

Des accords de libre-échange ont été conclus entre l'AELE et les 13 pays suivants (état en septembre 1998): Bulgarie, Estonie, Hongrie, Israël, Lettonie, Lituanie, Maroc, Pologne, Roumanie, Slovaquie, Slovénie, Tchéquie et Turquie. Des déclarations de coopération ont été signées avec plusieurs autres pays ou autorités: Albanie, Jordanie, Egypte, Liban, Macédoine, OLP, Tunisie. Ces déclarations ont à terme pour objectif la conclusion d'accords de libre-échange (négociations bien avancées avec la Tunisie, l'Egypte souhaitant terminer en priorité les négociations avec l'Union européenne). Des négociations en vue d'accords de libre-échange sont aussi en cours avec Malte et Chypre, et l'AELE souhaite aussi un accord de ce type avec le Conseil de coopération du Golfe (Emirats arabes, Oman et Arabie Saoudite). Les accords avec les pays Baltes (Estonie, Lettonie et Lituanie) ont été approuvés par les Chambres fédérales en 1997. Les accords existants sont régulièrement revus et adaptés aux développements intervenus dans la politique commerciale internationale (réglementations techniques, ouverture des marchés publics, protection des droits de propriété intellectuelle, services, investissements). De telles révisions ont concerné récemment la Bulgarie, Israël, la Roumanie et la Turquie. 
L'accord de libre-échange avec le Maroc a été signé le 19 juin 1997. La Suisse l'a ratifié en 1998. C'est le premier accord de ce type avec un pays de l'Afrique du Nord. Le Maroc représente le troisième marché d'exportation de la Suisse en Afrique (après l'Afrique du Sud et l'Egypte). L'accord couvre le secteur industriel, les produits agricoles transformés et les produits de la pêche. Il est asymétrique en ce sens que les Etats de 1'AELE éliminent complètement les droits de douane dès son entrée en vigueur, alors que le Maroc bénéficie d'une période transitoire de douze ans pour éliminer progressivement sa protection douanière. Le secteur agricole fait l'objet d'arrangements bilatéraux entre les pays de l'AELE et le Maroc (baisse des droits de douane sur certains produits n'allant pas au-delà de celles accordées aux autres partenaires libre-échangistes de la Suisse).

\section{Accords de commerce et de coopération économique}

Un accord de commerce et de coopération économique entre la Suisse et la République kirghize a été signé le 10 mai 1997; approuvé par les Chambres fédérales en 1998, cet accord est valable cinq ans et peut se proroger de cinq ans en cinq ans. Il a pour but de renforcer les relations économiques bilatérales entre les deux pays et doit permettre d'appuyer le processus de réformes engagé au Kirghizistan pour instaurer une économie de marché. L'accord s'appuie sur les principes fondamentaux de l'OMC. Les pays s'accordent le traitement de la nation la plus favorisée et renoncent à un traitement discriminatoire dans les échanges commerciaux. Les marchandises doivent bénéficier du traitement national. Les paiements liés au commerce doivent avoir lieu dans une monnaie convertible aux prix du marché (sans exiger le troc ou des marchés compensatoires). Les parties contractantes s'engagent à assurer une protection adéquate des droits de propriété intellectuelle (dans un délai de trois ans), sur la base des accords de l'OMC (ADPIC/TRIPS, Accord sur les aspects des droits de propriété intellectuelle liés au commerce /Agreement on Trade-related Aspects of Intellectual Property Rights), de la Convention de Paris sur la propriété industrielle, de la Convention de Berne (protection des œuvres littéraires et artistiques) et de la Convention de Rome (protection des artistes et producteurs de radio). Les Etats s'engagent à lutter contre la piraterie et la contrefaçon. La coopération économique sera favorisée pour consolider et diversifier les échanges, contribuer au développement de l'économie, accélérer les ajustements structurels (affermir la position de la République kirghize en matière de politique commerciale), promouvoir la participation des PME aux échanges et l'assistance technique dans le domaine de la propriété intellectuelle. Un comité mixte de représentants des parties contractantes est institué pour veiller à l'application de l'accord.

Le commerce entre la Suisse et le Kirghizistan est peu développé, avec quasiment pas d'importations de la Suisse en provenance de ce pays et des exportations de moins d'un million de francs en 1995. L'augmentation du commerce en 1996 est largement due aux livraisons de machines financées par le crédit d'aide aux pays de l'Europe centrale et orientale.

Des accords de commerce et de coopération économique existent déjà avec la Macédoine (entré en vigueur en 1996) et le Kazakhstan (entré en vigueur le $1^{\text {er }}$ juillet 1997). Des accords similaires ont été signés avec l'Arménie (signé en novembre 1998), la Fédération de Russie, l'Ukraine, l'Ouzbékistan, le Bélarus et la Moldavie. Un accord a aussi été paraphé avec la Géorgie en juin 1997 et un autre est en préparation avec le Turkménistan. 
Cet accord permettra à la Suisse d'aider le Vietnam à se doter d'un cadre légal et institutionnel en matière de propriété intellectuelle. L'accord a été adopté par le Parlement en 1998'. C'est le premier accord orienté exclusivement sur le domaine de la propriété intellectuelle, même si les accords de libre-échange ou les accords bilatéraux de commerce comprennent généralement aussi un volet sur la propriété intellectuelle.

Les parties contractantes s'engagent à assurer une protection effective et non discriminatoire des droits de propriété intellectuelle et à édicter à cette fin les dispositions légales nécessaires. Le Vietnam, qui n'est pas encore membre de l'OMC, s'engage par cet accord à assurer un niveau de protection au moins égal à celui des accords de l'Uruguay Round (accords ADPIC/TRIPS) d'ici au $1^{\text {er }}$ janvier 2000. La protection doit couvrir par exemple les droits d'auteur, les marques, les indications géographiques, les dessins et modèles industriels, les brevets d'invention et la protection des variétés végétales. Le traitement national implique que le ressortissant suisse doit bénéficier d'un traitement non moins favorable que celui qui est réservé aux Vietnamiens. La clause de la nation la plus favorisée doit empêcher que les Suisses ne soient traités moins favorablement que ceux de n'importe quel autre Etat. La Suisse s'engage à fournir un programme spécial de coopération technique d'une durée initiale de trois ans pour aider le Vietnam à renforcer les droits de propriété intellectuelle. Ce programme de coopération comprend des mesures pour adapter la législation nationale, la formation de personnel qualifié, la modernisation de l'infrastructure liée aux technologies de l'information. Il sera financé sur le crédit de programme pour la continuation du financement des mesures de politique économique et commerciale au titre de la coopération internationale au développement. Le programme comprend par exemple les mesures suivantes (extraits de l'annexe 2 de l'accord) :

- déterminer les besoins de modernisation du cadre juridique du Vietnam, proposer de nouvelles lois de protection des droits de propriété intellectuelle;

- former et qualifier le personnel dans les offices administratifs responsables de la propriété intellectuelle;

- moderniser les systèmes d'information en matière de brevets et fournir les équipements techniques nécessaires;

- attirer davantage l'attention du public sur l'importance de protéger les innovations et les créations, de lutter contre la contrefaçon et la piraterie;

- Encourager les entreprises, les communautés locales et les individus à faire usage des droits de propriété intellectuelle dans tous les domaines technologiques.

Ca ASTM 1998, p. 297 (dans la partie «Revue») et pp. 59-61 de l'article de T. Thu-Lang Tran «Vision et dialogue: les objectifs de la Suisse dans le système global de la propriété intellectuelle» (dans la partie «Analyses et positions»).

\section{$\square$ Accords économiques entre la Suisse et les pays de l'Est}

Le tableau suivant récapitule les informations sur les accords bilatéraux entre la Suisse et les pays de l'Europe centrale et orientale ainsi que les pays de la CEI.

7. «Message concernant l'Accord entre la Confédération suisse et la République socialiste du Vietnam sur la protection de la propriété intellectuelle et la coopération dans ce domaine» du 19 janvier 1998 , Feuille fédérale, ${ }^{\circ} 9,10.3 .98$ (annexe au «Rapport sur la politique économique extérieure $97 / 1+2 »$ ). 
Tableau n`17: Accords économiques entre la Suisse et les pays de l'Est (date d'entrée en vigueur) - état septembre 1998

\begin{tabular}{lcccc}
\hline & $\begin{array}{c}\text { Accords commerciaux } \\
\text { et de coopération }\end{array}$ & $\begin{array}{c}\text { Accords de } \\
\text { libre-échange }\end{array}$ & $\begin{array}{c}\text { Accords de protection } \\
\text { des investissements }\end{array}$ & $\begin{array}{c}\text { Accord double } \\
\text { imposition }\end{array}$ \\
\hline Albanie & 1996 & - & 1993 & en prép. \\
\hline Bosnie-Herzégovine & - & - & - & - \\
\hline Bulgarie & 1973 & 1994 & 1993 & 1993 \\
\hline Estonie & 1926 & 1997 & 1993 & en prép. \\
\hline Croatie & en prép. & - & 1997 & - \\
\hline Lettonie & 1925 & 1997 & 1993 & en prép. \\
\hline Lituanie & - & 1997 & 1993 & en prép. \\
\hline Macédoine & 1996 & - & 1997 & en prép. \\
\hline Pologne & 1973 & 1994 & 1990 & 1992 \\
\hline Roumanie & 1973 & 1994 & 1994 & 1994 \\
\hline Rép.féd. de Yougoslavie & - & - & - & - \\
\hline Slovaquie & 1971 & 1992 & 1991 & 1997 \\
\hline Slovénie & - & $1995^{* *}$ & 1997 & $1996^{*}$ \\
\hline Rép. tchèque & 1971 & 1992 & 1991 & 1996 \\
\hline Hongrie & 1974 & 1994 & 1989 & 1982 \\
\hline Arménie & en prép. & - & - & - \\
\hline Azerbaïdjan & en prép. & - & - & - \\
\hline Géorgie & en prép. & - & - & - \\
\hline Kazakhstan & 1997 & - & 1998 & 1988 \\
\hline Kirghizistan & 1998 & - & en prép. & - \\
\hline Moldavie & 1996 & - & 1996 & en prép. \\
\hline Russie & 1995 & - & 1991 & 1997 \\
\hline Tadjikistan & - & - & - & - \\
\hline Turkménistan & en prép. & - & - & - \\
\hline Ukraine & 1996 & - & 1997 & 1998 \\
\hline Ouzbékistan & 1994 & - & 1993 & en prép. \\
\hline Bélarus & 1994 & - & 1994 & en prép. \\
\hline Sourear & & & & \\
\hline
\end{tabular}

Source: annexe II du «Message sur la poursuite de la coopération renforcée avec l’Europe de l'Est et les pays de la CEI » du 19 août 1998, Feuille fédérale, no 44, 10.11.98 (message n98.049).

* Signature.

** Application provisoire.

\subsection{DIPLOMATIE COMMERCIALE}

Les visites des conseillers fédéraux dans les pays en développement comportent les volets de discussions économiques (les délégations suisses comprennent des représentants de l'économie privée), mais sont aussi souvent l'occasion de signer des accords bilatéraux et de visiter des projets de coopération au développement. Les délégations menées par les conseillers fédéraux permettent d'ouvrir des portes aux représentants suisses de l'économie privée dans les ministères des pays visités, pour faciliter les contacts à haut niveau dans le pays partenaire (occasion pour des entreprises suisses de rencontrer des premiers ministres, des ministres des Affaires extérieures, du Commerce et de l'Industrie, de l'Energie et des Télécommunications ou de l'Economie). 
Thaïlande/Malaisie

Pour son premier voyage officiel en dehors de l'Europe, le conseiller fédéral Pascal Couchepin s'est rendu dans ces pays début août 1998, à la tête d'une délégation mixte d'une trentaine de personnes comprenant des représentants de l'économie privée. La Thaillande fait partie des principaux pays en développement partenaires commerciaux de la Suisse ( $4^{\mathrm{e}}$ plus grand fournisseur de la Suisse en 1996 et $8^{\text {e }}$ principal marché d'exportation). En 1997, les importations en provenance de ce pays se sont élevées à 560 millions de francs, tandis que les exportations de la Suisse vers la Thaillande ont atteint 901 millions de francs (pour la Malaisie, les importations se sont élevées à 178 millions de francs et les exportations à 628 millions de francs en 1997). Au premier semestre de l'année 1998, les ventes suisses à destination de ces pays ont chuté d'un tiers. Le stock des investissements suisses en Malaisie s'élevait à 858 millions de francs en 1996, ce qui place la Suisse au $5^{\mathrm{e}}$ rang des investisseurs étrangers (investissements en Thaïlande de 692 millions de francs). En Thaïlande, les discussions ont porté sur les thèmes suivants: crise monétaire et financière, ambitieux programme de privatisation dans ce pays, révision de la loi sur les brevets. La ministre du Commerce et de l'Industrie de Malaisie a à nouveau rencontré Pascal Couchepin en octobre 1998, à Berne. Le conseiller fédéral a exprimé à cette occasion ses préoccupations à propos des mesures de contrôle des capitaux et des changes prises par le gouvernement malaisien.

\section{Emirats arabes unis/Koweït}

Une délégation économique menée par le secrétaire d'Etat Franz Blankart s'est rendue au Koweït et dans les Emirats arabes unis du 30 octobre au 6 novembre 1998, avec des représentants de l'industrie horlogère, des banques, de la chimie, de l'industrie alimentaire, de l'industrie des machines, de l'OSEC et de la Chambre arabo-suisse du commerce et de l'industrie. Des entretiens économiques ont eu lieu (notamment sur la question du piratage de montres et des copies de médicaments suisses) et des accords sur la protection des investissements ont été signés avec le Koweït et les Emirats arabes unis. Une convention de double imposition sera négociée avec les Emirats, et la Suisse aimerait conclure un accord de libre-échange entre l'AELE et le Conseil de coopération du Golfe. La région est un important marché d'exportation pour la Suisse: exportations pour un montant de 1 milliard 76 millions vers l'Arabie Saoudite en 1997, de 610 millions de francs vers les Emirats arabes unis, de 212 millions de francs vers le Koweït, de 156 millions de francs vers Oman, de 106 millions de francs vers le Bahreïn et de 82 millions de francs vers le Qatar.

\section{Inde}

Le conseiller fédéral Flavio Cotti s'est rendu en Inde fin novembre 1998, accompagné d'une délégation de représentants de l'administration fédérale et de l'économie privée suisse. Le volume commercial entre les deux pays est important et la Suisse fait partie des dix plus importants investisseurs en Inde. Les exportations suisses vers ce pays se sont élevées à 591 millions de francs en 1997 (surtout des machines-outils, appareils, machines textiles, machines à imprimer, produits chimiques et pharmaceutiques). Les importations en provenance de l'Inde s'élevaient la même année à 451 millions de francs (surtout des produits textiles). Outre les entretiens économiques, Flavio Cotti a visité certains projets de coopé- 
ration (production de frigos sans gaz CFC, projet de production de soie, programme de petits crédits). L'Inde est le principal pays bénéficiaire de la coopération suisse. La Suisse avait réduit de 5\% le budget de la coopération au développement pour l'Inde suite aux essais nucléaires indiens. Plusieurs divergences existent dans le domaine économique: la Suisse reproche à l'Inde d'avoir des barrières trop élevées à l'encontre des importations de produits textiles haut de gamme et de produits horlogers de luxe; de leur côté, les entreprises horlogères indiennes se plaignent de n'avoir qu'un accès très limité dans les foires commerciales suisses. L'Inde a par contre accéléré en automne 1998 la révision de la loi sur les patentes, pour se conformer d'ici à l'an 2005 aux accords ADPIC de l'OMC (étendre la protection par les brevets aux produits, et non plus seulement la concéder aux procédés) et autoriser les entreprises étrangères à déposer leurs demandes de brevets.

Ca ASTM 1998, dossier sur la propriété intellectuelle: pp. 25-46 (article de D.M. Nachane «Intellectual Property Rights and the WTO: A Southern Perspective) et pp. 95-104 (article de P. S. Mehta «TRIPS and Pharmaceuticals: Implications for India»).

\section{Autres visites de conseillers fédéraux ou de délégations économiques}

• Le conseiller fédéral Flavio Cotti a mené des discussions politiques en Jordanie et en Israël lors de son voyage au Proche-Orient de mai 1998.

- Réunion de la Commission mixte Suisse-Ouzbékistan en mars 1998, qui a permis un échange de vues et d'informations sur la situation économique, l'évolution des relations bilatérales et les moyens de développer les conditions-cadres nécessaires aux échanges et aux investissements. La quasi-totalité des importations de la Suisse en provenance de ce pays est composée par des produits textiles (6 millions de francs en 1997). Les exportations sont aussi assez modestes ( 23 millions de francs, surtout des produits agricoles, machines, produits chimiques et pharmaceutiques).

• Visite d'une délégation économique mixte en Iran en mars 1998, dirigée par l'ambassadeur Nicolas Imboden, avec des représentants de l'industrie des machines, de l'horlogerie, de la chimie, des banques et du commerce, et des bureaux d'ingénieurs de l'industrie alimentaire. La visite a été l'occasion de signer un accord de protection des investissements.

\section{AMÉRIQUE LATINE}

\section{Mexique}

Le conseiller fédéral Pascal Couchepin s'est rendu dans ce pays du 6 au 10 octobre 1998, à la tête d'une délégation comprenant une douzaine de représentants de l'économie suisse. Un mémorandum d'entente sur le commerce et la coopération économique a été signé à cette occasion. Cet arrangement couvre un programme de travail conjoint portant sur la libéralisation du commerce de biens et services et des investissements, sur le renforcement de la coopération économique et sur la promotion des échanges commerciaux et des investissements. Cet accord constitue un pas vers la création d'une zone de libre-échange entre l'AELE et le Mexique (ce pays entamant des discussions avec l'Union européenne sur un accord de libre-échange).

Les deux pays ont déjà signé une convention de double imposition en 1993 et un accord de promotion et de protection des investissements en 1995. Les exportations de la Suisse vers le Mexique se sont élevées à 634 millions de francs en 
1997 et comptaient principalement des machines (38\% des exportations), des produits chimiques $(37 \%)$, des produits pharmaceutiques, des montres, instruments et appareils. Les importations en provenance de ce pays s'élevaient à 68 millions de francs et comprenaient surtout des produits chimiques, des produits agricoles (dont $46 \%$ de café et $30 \%$ de miel) et des machines. Les investissements suisses au Mexique s'élevaient à 1845 millions de francs en 1997 (stock cumulé) ${ }^{8}$. Les entreprises suisses y emploient 20’300 personnes. Le Mexique est le deuxième plus important pays de destination des investissements suisses en Amérique latine.

\section{Chef d'Etat brésilien en Suisse}

Fernando Henrique Cardoso a entrepris une visite officielle en Suisse en janvier 1998. Le Parlement brésilien n'a pas encore ratifié l'accord de protection des investissements signé en 1994. La Suisse a aussi exprimé son désir de négocier une convention de double imposition avec le Brésil. Le Forum Suisse-Brésil, plate-forme d'ONG suisses soutenant des projets de coopération au Brésil, a transmis au président une demande pour que le Brésil respecte mieux les droits des enfants et les territoires des peuples autochtones, ainsi que l'application de la réforme agraire. Le Brésil est le principal partenaire commercial en Amérique latine et le plus important pays de destination des investissements suisses dans ce continent (investissements de 4,9 milliards de francs fin 1996).

\section{AFRIQUE}

\section{Afrique du Sud}

Le conseiller fédéral Flavio Cotti a effectué une visite officielle en Afrique du Sud du 2 au 6 août 1998, accompagné d'une délégation économique comprenant entre autres des représentants des entreprises Asea Brown Boveri, Ascom, Kuoni et Holderbank ainsi que de l'UBS. C'était la quatrième visite d'un conseiller fédéral depuis 1994; le président Nelson Mandela s'est déjà rendu en Suisse à quatre reprises.

L'Afrique du Sud représente le plus grand marché pour les exportations suisses vers le continent africain (exportations de 558 millions de francs en 1997). Elle est aussi, derrière le Nigeria, le deuxième pays plus grand fournisseur de ce continent (importations en provenance d'Afrique du Sud pour un montant de 395 millions de francs en 1997). La Suisse importe surtout des matières premières, des produits agricoles et du vin. Il faut toutefois remarquer que les importations d'or ne sont pas prises en compte dans les statistiques douanières. L'Afrique du Sud en est pourtant le premier producteur mondial et ce produit est en partie raffiné et vendu en Suisse. Si l'on tient compte du commerce d'or, la Suisse est le principal marché d'exportation de l'Afrique du Sud (devant les Etats-Unis et la Grande-Bretagne) $)^{9}$. La Suisse exporte surtout des machines (30\% des exportations), des produits chimiques et pharmaceutiques (35\% environ), des produits alimentaires.

La Suisse est le $6^{\mathrm{e}}$ investisseur étranger le plus important pour l'Afrique du Sud (derrière les USA, l'Allemagne, la Grande-Bretagne, la Malaisie et la Corée du Sud) ; 300 entreprises suisses sont présentes dans ce pays, pour des investisse-

8. Source des chiffres: BNS, Monnaie et conjoncture, Bulletin trimestriel n ${ }^{\circ} 4$, décembre 1997.

9. Neue Zürcher Zeitung, «Nelson Mandela in Zürich», 3.9.97. 
ments d'un montant total de 4 milliards de francs environ. Les entreprises suisses y emploient plus de $25^{\prime} 000$ personnes (dont près de 6900 par Nestlé) ${ }^{10}$. L'accord de promotion et de protection réciproque des investissements signé en 1995 est entré en vigueur le 29 novembre 1997.

\section{Zones d'ombre dans le passé des relations Suisse-Afrique du Sud}

Les relations économiques et politiques entre les deux pays comportent des zones d'ombre, notamment par le fait que la Suisse ne s'était pas associée au boycott international des relations avec le régime de I'apartheid (Nations Unies) et aux sanctions prises par certains Etats (Etats-Unis et Union européenne par exemple). Les importations en provenance de l'Afrique du Sud ont augmenté très fortement de 1986 à 1989, passant de 154 millions de francs à 1 milliard 178 millions de francs. Ce phénomène est dû en bonne partie aux considérables importations directes de diamants par une filiale de la maison sud-africaine De Beers. Les importations de diamants transitaient auparavant par la Grande-Bretagne, apparaissant ainsi dans les statistiques comme importations de ce pays.

La conseillère nationale Pia Hollenstein (écologiste/SG) a demandé en mars 1997 au Conseil fédéral d'ouvrir une enquête historique sur les relations entre la Suisse et le régime d'apartheid. Dans sa réponse, le Conseil fédéral estime qu'une telle enquête ne répond à aucune nécessité. II justifie aussi la politique du gouvernement suisse à l'égard de Pretoria*. En 1986, la Suisse s'était déclarée hostile à l'arme de la sanction économique. Elle défendait l'universalité des relations économiques suisses (pilier de la politique étrangère helvétique), tout en condamnant la politique d'apartheid. Le Conseil fédéral reconnaît dans sa réponse à l'interpellation parlementaire que si l'attitude de l'époque était compréhensible (la Suisse n'agirait pas forcément de la même manière aujourd'hui), "elle ne révélait politiquement pas une vision très large».

Une plate-forme d'ONG suisses préparent une campagne sur les pratiques des entreprises suisses pendant le régime de ségrégation raciale en Afrique du Sud. La campagne vise à faire la lumière sur l'attitude du secteur public et de l'économie privée pendant cette période et demande l'annulation de la dette contractée par l'ancien régime (les prêts et crédits accordés au régime d'apartheid étant considérées par les ONG comme illégitimes). Les populations sud-africaine ainsi que des pays voisins de l'Afrique du Sud (qui ont subi les actions de déstabilisation menées par le régime de l'apartheid) ne devraient pas payer deux fois le prix de l'apartheid en étant obligées de rembourser la dette ${ }^{* *}$. Les profits engrangés par les entreprises et les banques devraient selon les ONG être déclarés illégitimes, et ces entreprises ont un devoir de réparation envers les populations.

\footnotetext{
* Le Temps, "Rouvrir le dossier Suisse-Afrique du Sud ne répond à aucune nécessité», 20.7.98.

** Campagne "Crimes de l'apartheid: il est temps de réparer". Voir site Internet de la COTMEC (Commission Tiers Monde de l'Eglise catholique, tél. 022/329.26.81) : http://www.cath.ch/cotmec, rubrique "Actions". Informations aussi auprès d'Aktion Finanzplatz Schweiz Dritte Welt, Bâle, tél. 061/693.17.00. La campagne est (en juin 1998) déjà soutenue par une trentaine d'ONG suisses, une coalition d'ONG sud-africaines et une dizaine d'ONG en Allemagne.
}

La Suisse a apporté une aide de 50 millions de francs à l'Afrique du Sud de 1986 à 1994. Lors du premier voyage de Cotti (en 1994), un programme d'aide au développement d'un montant de 80 millions de francs avait été annoncé (19951999), notamment pour le financement de bourses d'études, de petits crédits, l'amélioration de la situation dans les townships (assainissement, approvisionnement en eau, amélioration des infrastructures de traitement des eaux usées et voirie dans l'agglomération de Port Elizabeth), le soutien à la démocratisation et la

10. Sources des chiffres: Neue Zürcher Zeitung, 3.8.98; BNS, Monnaie et conjoncture, Bulletin trimestriel nº décembre 1997. Les chiffres du stock des investissements publiés par la Banque nationale suisse parlent d'un montant de 1,1 milliard de francs investis, investissements en progression depuis 1994. 
promotion des droits de l'homme. Des contributions ont par exemple été versées pour la commission «Vérité et Réconciliation», ainsi que pour la préparation d'une nouvelle Constitution (1 million de francs pour chacun des deux projets) et pour l'appui du processus d'élection (en 1994). Un bureau de coordination de la DDC est établi à Johannesburg. Lors de son voyage de 1998, Flavio Cotti a visité certains projets soutenus par la Suisse (Port Elizabeth). Il a confirmé la poursuite de la coopération avec ce pays (2000-2004), avec un accent mis sur l'appui au développement du système de formation, la poursuite de l'amélioration de l'habitat à Port Elizabeth et le crédit pour des entrepreneurs privés.

\section{Côte d'Ivoire/Ghana}

Le secrétaire d'Etat Franz Blankart s'est rendu à Abidjan et à Accra entre fin septembre et début octobre 1998, à la tête d'une délégation économique mixte composée d'une quinzaine de représentants de l'économie suisse. Une déclaration de coopération concernant le désendettement et la gestion de la dette a été signée en Côte d'Ivoire. Un projet d'assistance technique établi conjointement par la CNUCED, le CCI et l'OMC a été signé avec les autorités ghanéennes et un représentant des agences multilatérales. Il devrait permettre au Ghana de mieux participer aux travaux de l'OMC et de faciliter l'intégration du pays dans le système commercial multilatéral.

\section{SOURCES}

«Rapport sur la politique économique extérieure 97/1+2 et messages concernant des accords économiques internationaux» du 19 janvier 1998, Feuille fédérale, nº $9,10.3 .98$, pp. 605-934 (message n 97.090).

Communiqués de presse des services d'information de l'administration fédérale, DFAE, «Visite officielle de Flavio Cotti en Afrique du Sud», 27.7.98.

Communiqués de presse des Affaires économiques extérieures, «Visite d'une délégation économique mixte en Iran», 3.3.98; «Réunion de la Commission mixte Suisse-Ouzbékistan», 6.3.98; «Mission économique du conseiller fédéral Pascal Couchepin au Mexique», 25.9.98; «Visite d'une délégation économique au Koweît et dans les Emirats arabes unis», 29.10.98.

BNS, «L'évolution des investissements directs en 1996», Monnaie et conjoncture, Bulletin trimestriel no 4, Banque nationale suisse, décembre 1997.

Flavio Cotti, «Wechselwirkungen zwischen der schweizerischen Aussenpolitik und Wirtschaftspolitik», Schweiz. Zeitschrift für Volkswirtschaft und Statistik, Vol. 132 (3), 1996, S. 293-298.

Philippe Gugler, «Les défis pour la politique économique extérieure suisse dans le nouveau contexte contexte économique mondial», Schweiz. Zeitschrift für Volkswirtschaft und Statistik, Vol. 132 (3), 1996, S. 299-316.

La Liberté, 28.7.98 (Flavio Cotti en Afrique du Sud et Pascal Couchepin en Thaïlande) ; 20.11.98 (Flavio Cotti en Inde).

Le Temps, «Afrique du Sud: un dossier brûlant», 18.6.98; «Rouvrir le dossier Suisse-Afrique du Sud ne répond à aucune nécessité», 20.7.98; «La Suisse veut renforcer ses liens économiques avec l'Afrique du Sud», 5.8.98; «Le Mexique demeure attractif pour les Suisses», 12.10.98.

Neue Zürcher Zeitung, «Ein Gespräch mit dem brasilianischen Präsidenten Cardoso», 30.1.98; «Ausbau der Beziehungen zu Südafrika», 3.8.98; «Bundesrat Couchepin in Thailand», 4.8.98; «Cotti: Wir haben Vertrauen in Südafrika», 5.8.98; «Malaysia umwirbt Schweizer Investoren», 7.8.98; «Bundesrat Couchepin als Türöffner in Mexiko», 12.10.98; «Investitionsschutzabkommen mit Golf-Emiraten», 5.11.98; «Staatsbesuch von Bundespäsident Cotti in Indien», 23.11.98; «Dichtes Gesprächsprogramm in Delhi. Abschluss des Cotti-Besuchs», 25.11.98.

SITE INTERNET

Office fédéral des affaires économiques extérieures: http://www.admin.ch/bawi/index.html 